\title{
PILOT TRIAL OF STEPPED CARE CBT FOR PEDIATRIC OCD
}

\author{
Adam Lewin, \& Omar Rahman \\ University of South Florida (USA)
}

\begin{abstract}
Introduction: Obsessive Compulsive Disorder (OCD) affects 1-2\% of youth, with $75 \%$ of those having a comorbid disorder. OCD is typically impairing and disruptive to social, academic, and family functioning. Impairment related to OCD usually compounds over time and leads to significant distress, impairment, and dysfunction.

Evidence-based treatment for OCD includes cognitive behavioral therapy with exposures and response prevention (CBT-ERP) and selective seratonin reuptake inhibitors (SSRIs). These are often used in combination. However, for pediatric OCD, CBT-ERP is the preferred first line of treatment, with SSRI recommended for severe cases, non-responders to CBT, and for those where CBT is not available or acceptable.

Despite this, CBT-ERP has limited accessibility and dissemination for several reasons: relatively few trained experts, long waitlists, distance and travel time, requirement of 12-14 visits, and expenses (treatment, missed work, etc.). As a result, many youth do not receive the recommended treatment. Bridging this gap between research knowledge and implementation of good treatment is a primary concern.

We investigated an alternate treatment approach, Stepped Care CBT (SSCBT), to address the above concern. SSCBT has the advantage of allowing for larger caseloads for therapists, shorter wait times for patients, decreased life disruption, and decreased cost. The primary question was this: Can a parent-delivered, therapist-assisted CBT mitigate the need for full course, therapist-directed treatment?

Method: Forty-five youth were screened for the study. Thirty-four youth with OCD were randomized at a $2: 1$ ratio into SSCBT $(n=22)$ or standard therapy $(n=12)$. SSCBT consisted of Step One: a low therapist-intensity treatment, consisting of 3 clinic sessions with therapist, six weekly phone contacts, and 11 parent-child meetings guided by a workbook; and Step Two: for non-responders in Step One, nine sessions of therapist-directed standard in-clinic CBT-ERP. Standard care consisted of 12 sessions of in-clinic, therapist directed CBT-ERP.

Results: In the standard group, the treatment response rate was 83\%. In SSCBT, the response rate was 79\% after Step One and 93\% after Step Two.

Discussion: The results provide support for the stepped care model as applied to CBT for OCD in youth. This has the potential to make effective treatment available to more youth, and at a significantly reduced cost and disruption. We also discuss secondary findings from the study, as well as strength, limitations, and suggestion for future research.
\end{abstract}

Keywords: Obsessive compulsive disorder, cognitive behavioral therapy, exposure and response prevention, stepped care, therapy. 